


\section{IRA BERLIN \\ (NEW YORK, 1941 - WASHINGTON DC, 2018)}

Keila Grinberg ${ }^{1}$

I

ra Berlin costumava ficar um bom tempo de olhos fechados durante suas aulas. No começo, achávamos que ele dormia enquanto nós, os alunos, discutíamos os textos; até entendermos que, quem sabe de propósito, ele estivesse nos deixando à vontade para falar livremente. Ou talvez dormisse mesmo, já antevendo, anos-luz à nossa frente, onde a argumentação nos levaria. $\mathrm{O}$ fato é que, faltando cerca de meia hora para o final da aula, ele invariavelmente perguntava: afinal, o que aprendemos com esse debate? E seguia resumindo nossas opiniões, desafiando-nos com visões alternativas, criticando os modismos acadêmicos e, quase sempre, nos brindando com uma impressionante síntese sobre o tema que estivéssemos estudando.

Um dos maiores historiadores da escravidão norte-americana de sua geração, Ira Berlin foi, no melhor sentido do termo, um mestre da síntese. Seus livros versam sobre praticamente todos os aspectos da história da escravidão nos Estados Unidos e da vida dos afro-americanos no pós-abolição. Não é exagero afirmar que ele transformou a historiografia norte-americana, conferindo à escravidão o papel central que de fato desempenhou na história dos Estados Unidos.

Ira Berlin nasceu em Nova York em 1941. Oriundo de família de imigrantes judeus da Bessarábia, atual Moldávia, cresceu no Bronx, de

1 Doutora em História pela Universidade Federal Fluminense, professora do Departamento de História da Universidade Federal do Estado do Rio de Janeiro. keila.grinberg@unirio.br. 
onde vinha seu inconfundível sotaque. Fez toda a sua formação acadêmica na University of Wisconsin, Madison, onde se graduou em Química em 1963 e defendeu o doutorado em História em 1970. Após uma breve passagem pela University of Illinois, em Chicago, e pelo Federal City College, em Washington, em 1974 tornou-se professor do Departamento de História da University of Maryland, onde, mesmo depois de sua aposentadoria, continuou dando aulas e frequentando seu espaçoso escritório no Francis Scott Key Hall. Nos últimos anos, além de dirigir o Center for Global Migration Studies, que fundou em 2011, ele dedicou-se intensamente à criação, na universidade, do memorial em homenagem ao abolicionista Frederick Douglass (1818-1895), a quem chamou de "a mais importante pessoa nascida em Maryland". ${ }^{2}$ Para seu deleite, a praça inaugurada em 2015, em cujo centro está a estátua de Douglass, rapidamente se transformou no local por excelência dos encontros do movimento Black Lives Matter (Vidas Negras Importam). ${ }^{3}$

Berlin começou a se interessar pelo estudo da história dos africanos e seus descendentes nos Estados Unidos ao marchar por direitos civis e se engajar na luta por igualdade racial nos anos 1960. Como muitos de sua geração, ele acreditava ser possível vencer o racismo através da aprendizagem sobre a escravidão, suas origens e sua conexão com a questão da raça. Estudando, "o problema estaria resolvido. Nós éramos extremamente ingênuos", declarou. ${ }^{4}$ A questão da conexão entre raça e escravidão o perseguiria por toda sua vida acadêmica. "A escravidão é o marco zero das relações raciais", ele escreveria muitos anos depois,

2 Harrison Smith, "Ira Berlin, Transformative Historian of Slavery in America, Dies at 77", The Washington Post, Washington, DC, 6 jun. 2018, <https://www.washingtonpost.com/local/ obituaries/ira-berlin-transformative-historian-of-slavery-in-america-dies-at-77/2018/06/06/ eddb33fc-6994-11e8-bf8cf9ed2e672adf_story.html?utm_term=.3dc9f1756336>, acessado em 11/02/2019 (tradução nossa).

3 Julie Greene, "Ira Berlin (1941-2018): Historian of Slavery and Freedom", Perspectives on History, Washington, DC, $1^{\mathrm{o}}$ out. 2018, <https://www.historians.org/publications-and-directories/perspectives-on-history/october-2018/ira-berlin-(1941\%E2\%80\%932018)>, acessado em 13/02/2019.

4 'Probably we all thought that once we've figured this all out, this business of race, once we've learned something about slavery and its origins, its connection to race, well, we could all go home early that night. The problem would be solved. We were extremely naïve". Neil Genzlinger, "Ira Berlin is Dead at 77; Groundbreaking Historian of Slavery", The New York Times, New York, 8 jun. 2018, <https://www.nytimes.com/2018/06/08/obituaries/ira-berlin-groundbreaking-historian-of-slavery-dies-at-77.html>, acessado em 13/02/2019 (tradução nossa). 
ressaltando o muito que havia também a aprender sobre a "desconcertante conexão entre escravidão e raça e sua relação com os espinhosos problemas de raça e classe no século XXI". 5

As associações entre os diferentes tempos e contextos seria uma das marcas de sua obra. Seu primeiro livro, Slaves Without Masters: The Free Negro in the Antebellum South (1974), baseado em sua tese de doutorado, é pioneiro na historicização da experiência dos negros livres e libertos no Sul dos Estados Unidos. ${ }^{6}$ Ao argumentar que as vidas dos libertos e seus descendentes variavam de acordo com a sua situação econômica, a época e a região onde moravam, e que estas distinções afetariam as comunidades negras por muito tempo após o fim da escravidão, ele demonstrava o quanto eram variáveis as experiências de escravidão e liberdade. ${ }^{7}$

Se hoje em dia esta afirmação parece óbvia, e evidentemente aplicável a toda parte onde houve escravidão no mundo atlântico, é porque historiadores como Ira Berlin se dedicaram a demonstrar que a escravidão não era uma instituição monolítica, na qual escravizados desempenhavam sempre o mesmo papel, trabalhando nas grandes plantations (no caso dos Estados Unidos, de algodão). Em Many Thousands Gone: The First Two Centuries of Slavery in North America (1998), seu livro mais conhecido e premiado, ele novamente abordaria a complexidade da escravidão norte-americana, desta vez a partir da lente da história do trabalho, demonstrando como, nos séculos XVII e XVIII, a instituição foi sendo definida local e regionalmente, tanto por senhores quanto por escravos. ${ }^{8}$ Estas diferenças teriam afetado o que Berlin denominou genericamente de "a cultura negra", ou seja, as formas de resistência, a religião, os hábitos de trabalho e a vida familiar dos escravizados e seus

5 Ira Berlin, "Coming to Terms with Slavery in Twenty-First-Century America", in James Oliver Horton e Lois E. Horton (eds.), Slavery and Public History: The Tough Stuff of American Memory (Chapel Hill: The University of North Carolina Press, 2006), p. 3 (tradução nossa).

6 O livro ganhou o prêmio de Best First Book da National Historical Society dos Estados Unidos.

7 Ira Berlin desenvolveu especificamente estas ideias no artigo "Time, Space, and the Evolution of Afro-American Society on British Mainland North America", American Historical Review, v. 85, n. 1 (1980), pp. 44-78.

8 O livro recebeu vários prêmios, entre os quais o Bancroft Prize, da Columbia University; o Elliott Rudwick Prize, da Organization of American Historians; e o Frederick Douglass Prize, do Gilder Lehrman Institute of American History, da Yale University. 
descendentes. Ira Berlin daria continuidade ao tema, alargando seu escopo temporal, em Generations of Captivity: A History of African-American Slaves (2003), o único de seus livros traduzido para o português, no qual estende sua análise até a Guerra Civil e a abolição, e em The Making of African America: The Four Great Migrations (2010), que inclui a chamada great migration do século XX, do Sul para o Norte dos Estados Unidos, concluindo com as migrações globais da passagem do século XX para o XXI. ${ }^{9}$

Estas quatro sínteses compõem o escopo básico da obra de Ira Berlin; e é aqui que, para além da historicidade e da complexidade da escravidão, encontramos desenvolvidos alguns de seus conceitos mais caros: o de sociedade escravista (em oposição a sociedade com escravos) e o de geração. Partindo do clássico estudo de Moses Finley, Escravidão antiga e ideologia moderna,${ }^{10}$ Berlin considera a distinção entre sociedades escravistas e sociedades com escravos como um dos melhores instrumentos conceituais para se analisar a escravidão nas Américas, desde que não seja usada para advogar alguma suposta benevolência das sociedades com escravos ou para construir argumentos a-históricos. Muito pelo contrário: o que interessa Berlin, especialmente em Many Thousands Gone, é justamente o processo de transformação dos Estados Unidos de uma sociedade com escravos para uma sociedade escravista. Este processo, evidentemente, nada tinha de linear: um dos pontos mais importantes de sua análise é justamente a dupla caracterização da Revolução Americana (1776), que perturbou a ordem escravista ao mesmo tempo que permitiu a expansão da escravidão sobre novas bases. Como bem enfatizou Eric Foner em texto em homenagem ao amigo, Berlin era mestre em lembrar que a "História não se move necessariamente em linha reta"."

9 Generations of Captivity foi publicado em português com o título Gerações de cativeiro: uma história da escravidão nos Estados Unidos (Rio de Janeiro: Record, 2006). O livro recebeu os prêmios Albert J. Beveridge Award, da American Historical Association; e Anisfield-Wolf Book Award, da Cleveland Foundation. Ira Berlin ainda receberia, pelo conjunto da obra, a medalha W. E. B. Dubois da Harvard University em 2014 e, no ano seguinte, o Award for Scholarly Distinction da American Historical Association.

10 Moses Finley, Escravidão antiga e ideologia moderna, São Paulo: Paz e Terra, 1991. Ver também Joyce E. Chaplin, "Berlin's Two Concepts of Slavery", Reviews in American History, v. 27, n. 2 (1999), pp. 188-193.

11 Eric Foner, "Ira Berlin, 1941-2018”, The Nation, New York, 8 jun. 2018, <https://www. thenation.com/article/ira-berlin-1941-2018/>, acessado em 12/02/2019 (tradução nossa). 
Esta caracterização mais ampla da sociedade norte-americana - se escravista ou com escravos - compõe o terreno a partir do qual Berlin desenvolveu seu conceito de geração: mais do que unidades de tempo, as gerações representam diferentes momentos na história da escravidão norte-americana, que poderiam ocorrer em diferentes épocas, de acordo com o lugar. Assim, em Gerações de cativeiro ele completa seu esquema explicativo definindo cinco diferentes gerações: a da travessia do Atlântico, a da plantation, a revolucionária, a da migração e, por último, a da liberdade (estas duas últimas seriam ainda mais desenvolvidas em The Making of African America).

Particularmente poderosa é sua descrição dos "crioulos atlânticos", membros da geração da travessia, homens e mulheres africanos cosmopolitas que, chegando à América do Norte mais ou menos ao mesmo tempo em que os primeiros colonizadores europeus, tinham vasto conhecimento do mundo atlântico e das variadas culturas e línguas que o formavam. Muitos deles haviam transitado pelo Atlântico, adquirindo competências desconhecidas dos europeus. Para Berlin, as habilidades dos crioulos atlânticos permitiram que alguns conquistassem a alforria e passassem a manejar o sistema com alguma autonomia. Publicado inicialmente como artigo na revista The William and Mary Quarterly(1996), o texto foi traduzido para o português em 2004, tendo sido de particular influência na historiografia atlântica sobre escravização e circulação de africanos no universo luso-afro-brasileiro dos séculos XVI e XVII. ${ }^{12}$

Em 1976, Ira Berlin criou, na University of Maryland, o Freedmen and Southern Society Project, do qual foi diretor até $1991 .{ }^{13}$ Como

12 Ira Berlin, "From Creole to African: Atlantic Creoles and the Origins of African-American Society in Mainland North America", The William and Mary Quarterly, v. 53, n. 2 (1996), pp. 251-288. Em português, o artigo foi publicado como "De crioulo a africano: as origens atlânticas da sociedade afro-americana na América do Norte Continental", Estudos Afro-Asiáticos, Rio de Janeiro, ano 26, n. 2 (2004), pp. 241-256. Um bom exemplo da influência do conceito de "crioulo atlântico" é o livro de Jane Landers, American Creoles in the Age of Revolutions, Cambridge: Harvard University Press, 2010; para uma discussão sobre o conceito no Atlântico português, ver Roquinaldo Ferreira, “'Ilhas crioulas': o significado plural da mestiçagem cultural na Africa atlântica", Revista de História, n. 155 (2006), pp. 17-41.

13 "Freedmen and Southern Society Project", <http://www.freedmen.umd.edu/index.html>, acessado em 17/10/2019. O projeto recebeu por duas vezes o Thomas Jefferson Prize da Society for History in the Federal Government, assim como o J. Franklin Jameson Prize da American Historical Association e o Abraham Lincoln Prize do Institute of Gettysburg College. 
declarou seu filho em uma entrevista, ele lia fontes a esmo no National Archives quando entrou na sala onde ficavam guardados os documentos do Bureau of Refugees, Freedmen and Abandoned Lands, ou simplesmente Freedmen's Bureau. ${ }^{14}$ Criada em 1865 para facilitar a integração da população pobre do Sul no pós-Guerra Civil, a agência de início fornecia alimentos, habitação e assistência médica; depois, criou escolas, forneceu assistência jurídica e ajudou principalmente os libertos a se estabelecer em terras confiscadas ou abandonadas durante a guerra no Sul. Embora tivesse sido criado para ser temporário e tivesse enfrentado inúmeras dificuldades de operacionalização, além da oposição de setores conservadores, o Bureau só foi extinto em 1872.

O que Ira Berlin encontrou foi uma mina de ouro, sonho dos historiadores. Os documentos que compunham o Bureau eram contados às centenas de milhares. Arquivados depois do fechamento da agência, eles permaneceram praticamente intocados por mais de cem anos. Sob sua direção, o projeto compilou, transcreveu, resumiu e publicou cerca de 50 mil documentos, em uma série de volumes denominada Freedom: A Documentary History of Emancipation, $1861-1867 .{ }^{15}$ É impossível dimensionar quantos estudos se basearam nesta documentação desde então, fornecendo novos dados e interpretações sobre a história da Guerra Civil americana (1861-1865) e da chamada Reconstrução, que a ela se seguiu (1865-1877).

O mesmo olhar para os arquivos - e a atenção às memórias da última geração de escravizados do país - levou Ira Berlin a dirigir a edição e publicação das entrevistas concedidas por libertos nos anos 1930 a pesquisadores do Federal Writers' Project, agência criada durante o New Deal que empregou, entre outros, historiadores, arquivistas e fotógrafos. Estas entrevistas foram organizadas e arquivadas na Library of Congress,

14 Smith, "Ira Berlin".

15 Freedmen \& Southern Society Project, Freedom: A Documentary History of Emancipation, 1861-1867, Chapel Hill: The University of North Carolina Press, 1985-. Até o presente, foram publicados seis volumes da série, além de outros quatro relativos a temas específicos, como família escrava e a experiência dos soldados negros durante a Guerra Civil. Ver "Publications of the Freedmen and Southern Society Project", <http://www.freedmen.umd.edu/fssppubs. htm>, acessado em 13/02/2019. 
em Washington. ${ }^{16}$ Como seria de esperar, a experiência de ouvir as vozes das pessoas que vivenciaram a escravidão foi impactante. Remembering Slavery, publicado em 1998, foi fundamental não só para a divulgação de canções escravas até então desconhecidas de pesquisadores, mas também por atrair a atenção do grande público, gerando documentários, leituras coletivas e peças teatrais, principalmente por parte daqueles que buscavam conhecer mais o seu próprio passado. ${ }^{17}$

"História não é sobre o passado; é sobre os argumentos que elaboramos sobre o passado. E porque é sobre os argumentos que elaboramos, é sobre nós mesmos". Com esta frase, Ira Berlin abriu seu último livro, The Long Emancipation: The Demise of Slavery in the United States. ${ }^{18}$ A importância da reflexão sobre a forma como o tema da escravidão impactava o presente foi relevante desde o início de sua carreira e só se fortaleceu ao longo do tempo. Assim, não é de se espantar que ele tenha sido pioneiro também no campo da História Pública. Ira Berlin colaborou em documentários e filmes de ficção, a exemplo de Doze anos de escravidão (2013), participou da organização de exposições, como Slavery in New York, aberta em 2005 na New York Historical Society, e foi um dos principais consultores do monumental National Museum of African American History \& Culture, inaugurado em 2016 em Washington, cuja exposição permanente é inspirada em seu trabalho. ${ }^{19}$

Mesmo sem a suposta ingenuidade do início da carreira, ele nunca deixou de insistir na importância de se lidar com o passado escravista, a "mais persistente contradição da história norte-americana", em um país obcecado com a liberdade. Daí seu interesse pelo ensino de História. Se "escravidão foi violência e imposição, se foi morte, foi também vida", escreveu, lembrando que os que foram escravizados "se recusaram a

16 Ver, a respeito, Jerrold Hirsch, Portrait of America: A Cultural History of the Federal Writers' Project, Chapel Hill: University of North Carolina Press, 2003; Library of Congress, "New Deal Programs: Selected Library of Congress Resources", <https://www.loc.gov/rr/program/ bib/newdeal/fwp.html>, acessado em 14/02/2019.

17 Para as repercussões do projeto ver, por exemplo, o filme Unchained Memories: Readings from the Slave Narratives (2003).

18 Ira Berlin, The Long Emancipation: The Demise of Slavery in the United States, Cambridge: Harvard University Press, 2015, p. 1 (tradução nossa).

19 Lonnie G. Bunch, "Statement on the Passing of Historian Ira Berlin", <https://nmaahc.si.edu/ about/news/statement-passing-historian-ira-berlin>, acessado em 11/02/2019. 
ser desumanizados pelo tratamento desumano" que receberam (tradução nossa). ${ }^{20}$ Ele considerava fundamental que esta mensagem chegasse aos estudantes de todas as escolas. Não que acreditasse que ela seria suficiente para vencer o racismo contemporâneo - não era -, mas porque era condição necessária para tal.

É possível que a última publicação de Ira Berlin, a tradução do primeiro capítulo de The Long Emancipation, tenha sido justamente em português..$^{21}$ Acho que ele teria gostado de saber disso. Ira mantinha estreitas relações com os historiadores brasileiros e gostava de vir ao Brasil. Salvo engano, visitou o país quatro vezes. A primeira foi em 1988, quando do centenário da abolição da escravidão no país; a segunda, dez anos depois, para participar de um seminário na Universidade Federal Fluminense (UFF), instituição com a qual a University of Maryland mantinha convênio. Ainda esteve em Florianópolis, em 2007, onde deu a conferência "Repensando escravidão e raça nos Estados Unidos e suas implicações para a história da escravidão e do racismo no mundo atlântico", que encerraria o III Encontro de Escravidão e Liberdade no Brasil Meridional, e na Bahia, em 2011, para proferir palestra e participar de reuniões da linha de pesquisa Escravidão e Invenção da Liberdade, na Universidade Federal da Bahia.

Ira Berlin foi a estrela principal do seminário de Niterói na UFF. Ao final, o professor escalado para debater com ele limitou-se a observar, algo irritado: "depois de ter estado aqui há dez anos, o senhor nos brinda com mais do mesmo; o que fez este tempo todo?". Foram momentos de tensão no auditório. Ao ouvir a tradução, Ira deu uma gargalhada. A resposta foi longa. "Espero ter trabalhado o suficiente para ser novamente convidado no futuro", finalizou, brincando. Sua elegância, generosidade e bom humor eram tão raros quanto a qualidade da sua obra. Ira Berlin fará muita falta.

20 Berlin, "Coming to Terms", p. 6.

21 Ira Berlin, "A quase centenária falência da escravidão nos Estados Unidos", in Ivana Stolze Lima, Keila Grinberg e Daniel Aarão Reis Filho (orgs.), Instituições nefandas: o fim da escravidão e da servidão no Brasil, nos Estados Unidos e na Rússia, Rio de Janeiro: Fundação Casa Rui Barbosa, 2018, p. 371-390. 


\section{Bibliografia selecionada de Ira Berlin - ordem cronológica}

Slaves Without Masters: The Negro in the Antebellum South, New York: Pantheon, 1974.

com Ronald Hoffman (eds.), Slavery and freedom in the age of the American Revolution, Charlottesville: University Press of Virginia, 1983.

com Barbara Fields, Thavolia Glymph, Joseph Reidy, e Leslie Rowland (eds.), The Destruction of Slavery, Cambridge: Cambridge University Press, 1985. (Freedom: A Documentary History of Emancipation, 1861-1867, série 1, vol. 1)

com Herbert Gutman (eds.), Power and Culture: Essays on the American Working Class, Pantheon: New York, 1987.

com Philip Morgan (eds.), The Slaves'Economy: Independent Production by Slaves in the Americas, Portland: Frank Cass, 1991.

com Thavolia Glymph, Steven Miller, Joseph Reidy, Leslie Rowland e Julie Saville (eds.), The Wartime Genesis of Free Labor: The Lower South, Cambridge: Cambridge University Press, 1991. (Freedom: A Documentary History of Emancipation, 1861-1867, série 1, vol. 3)

com Barbara Fields, Steven Miller, Joseph Reidy e Leslie Rowland (eds.), Slaves no More: Three Essays on Emancipation and the Civil War, Cambridge: Cambridge University Press, 1992.

com Barbara Fields, Steven Miller, Joseph Reidy e Leslie Rowland (eds.), Free at Last: A Documentary History of Slavery, Freedom, and the Civil War, New York: New Press, 1992.

com Steven Miller, Joseph Reidy e Leslie Rowland (eds.), The Wartime Genesis of Free Labor: The Upper South, Cambridge: Cambridge University Press, 1993. (Freedom: A Documentary History of Emancipation, 1861-1867, série 1, vol. 2)

com Philip Morgan (eds.), Cultivation and Culture: Labor and the Shaping of Slave Life in the Americas, Charlottesville: University of Virginia Press, 1993. 
com Leslie Rowland (eds.), Families and Freedom: A Documentary History of African-American Kinship in the Civil War Era, New York: New Press, 1997.

com Joseph Reidy e Leslie Rowland (eds.), Freedom's Soldiers: The Black Military Experience in the Civil War, Cambridge: Cambridge University Press, 1998.

Many Thousands Gone: The First Two Centuries of Slavery in North America, Cambridge: The Belknap Press of Harvard University Press, 1998.

com Marc Favreau e Steven Miller (eds.), Remembering Slavery: African Americans Talk About Their Personal Experiences of Slavery and Emancipation, New York: The New Press, 1998.

Generations of Captivity: A History of African American Slaves, Cambridge: Harvard University Press, 2003. (Gerações de Cativeiro: uma história da escravidão nos Estados Unidos, Rio de Janeiro: Record, 2006).

com Leslie Harris (eds.), Slavery in New York, New York: The New Press, 2005.

The Making of African America: The Four Great Migrations, New York: Viking, 2010.

The Long Emancipation: The Demise of Slavery in the United States, Cambridge: Harvard University Press, 2015. 